\title{
Alterations of immune cell subsets in relapsed, thymoma-associated minimal change disease: A case report
}

\author{
HELEN GHARWAN $^{1 *}$, YUSUKE TOMITA ${ }^{2 *}$, MIN-JUNG LEE $^{2}$, ANISH THOMAS $^{1}$, \\ ARLENE BERMAN $^{1}$, GIUSEPPE GIACCONE ${ }^{3}$, JANE TREPEL ${ }^{2}$ and ARUN RAJAN ${ }^{1}$
}

\author{
${ }^{1}$ Thoracic and Gastrointestinal Oncology Branch, and ${ }^{2}$ Developmental Therapeutics Branch, National Cancer Institute, \\ National Institutes of Health, Bethesda, MD 20892-1906; ${ }^{3}$ Department of Oncology, Lombardi Comprehensive Cancer Center, \\ Georgetown University, Washington, DC 20007, USA
}

Received November 7, 2014; Accepted May 20, 2015

DOI: $10.3892 / 01.2015 .3325$

\begin{abstract}
The most frequently described glomerulopathy in patients with thymoma is minimal change disease (MCD). The present study reports the case of a 63-year-old female with recurrent thymoma and poorly-controlled paraneoplastic MCD, who was enrolled on a phase I/II clinical trial (no. NCT01100944) and treated with the histone deacetylase inhibitor, belinostat, in combination with cisplatin, doxorubicin and cyclophosphamide. Treatment resulted in a complete radiological response, a dramatic reduction in proteinuria and changes in immune cell subset composition, consisting of a reduction in the number of $\mathrm{T}$ helper (Th)1, Th2, Th17 and regulatory $\mathrm{T}$ cells. Changes in $\mathrm{T}$-cell polarization were also observed with an increase in the Th1/Th2 ratio. To the best of our knowledge, the current study is the first to provide a detailed description of changes in immune cell subset composition in thymoma-associated MCD. Early administration of effective antitumor therapy should be considered in these cases, particularly when proteinuria is poorly controlled despite the use of steroids and other immunosuppressive therapies.
\end{abstract}

\section{Introduction}

Minimal change disease (MCD) is a well-described glomerulopathy that accounts for 10-15\% of primary nephrotic syndrome cases in adults (1). It is characterized by nephrotic-range proteinuria, edema, hypoalbuminemia, and hyperlipidemia. Biopsy findings include an absence of glomerular lesions

Correspondence to: Dr Arun Rajan, Thoracic and Gastrointestinal Oncology Branch, National Cancer Institute, National Institutes of Health, 10 Center Drive, Building 10, Room 12N226, Bethesda, MD 20892-1906, USA

E-mail: rajana@mail.nih.gov

\section{${ }^{*}$ Contributed equally}

Key words: thymoma, proteinuria, nephrotic syndrome, minimal change disease, T-cells on light microscopy and effacement of foot processes on electron microscopy. Steroids are used for first-line therapy of MCD. Immunomodulatory drugs such as cyclosporine, tacrolimus and mycophenolate mofetil are used for treatment of relapsed disease or in cases of steroid-resistance or steroiddependence $(2,3)$. MCD is also the most common cause of paraneoplastic glomerulonephritis in patients with thymoma, and T-cell dysfunction is considered to play an important role in its pathogenesis (4). However, the impact of antitumor or immunosuppressive therapy on the immune system in patients with thymoma-associated MCD is poorly understood. The present study describes changes in immune cell subset composition in response to tumor-directed therapy in a patient with relapsed, thymoma-associated MCD, who achieved a complete radiological tumor response and durable reduction in proteinuria.

\section{Case report}

A 63-year-old female with Masaoka stage IVA, World Health Organization type B2 thymoma $(5,6)$ was referred for treatment of recurrent thymoma 3 years after the initial diagnosis. Initial treatment consisted of surgical resection. The patient had developed anasarca and acute kidney injury 7 months before presentation with recurrent thymoma. A renal biopsy showed no global sclerosis on light microscopy and diffuse foot process effacement on electron microscopy. These changes were consistent with MCD. Oral prednisone was administered at a dose of $80 \mathrm{mg} /$ day, which was decreased to $20 \mathrm{mg} /$ day after 1 month due to steroid-induced myopathy. Despite an initial reduction in proteinuria, $\mathrm{MCD}$ relapse was observed within 4 months (Fig. 1). Cyclosporine was then administered; however, the patient remained symptomatic with fatigue and dyspnea on exertion. Upon presentation, medications included prednisone (5 mg/day) and cyclosporine (100 mg, orally, twice daily). Physical examination revealed pitting pedal edema up to the knees. Laboratory tests demonstrated hypoalbuminemia (serum albumin level, $2.3 \mathrm{~g} / \mathrm{dl}$; normal range, 3.5-5.2 g/dl), normal serum creatinine levels $(0.8 \mathrm{mg} / \mathrm{dl})$, proteinuria (urine protein excretion, $2.7 \mathrm{~g}$ in $24 \mathrm{~h}$; normal range, $30-150 \mathrm{mg}$ in $24 \mathrm{~h}$ ) and an elevated urine protein-creatinine ratio of $5.9 \mathrm{mg} / \mathrm{mg}$ (normal range, $0.001-0.16 \mathrm{mg} / \mathrm{mg}$ ). A computed tomography (CT) scan of the chest revealed a right paracardiac mass and multiple 


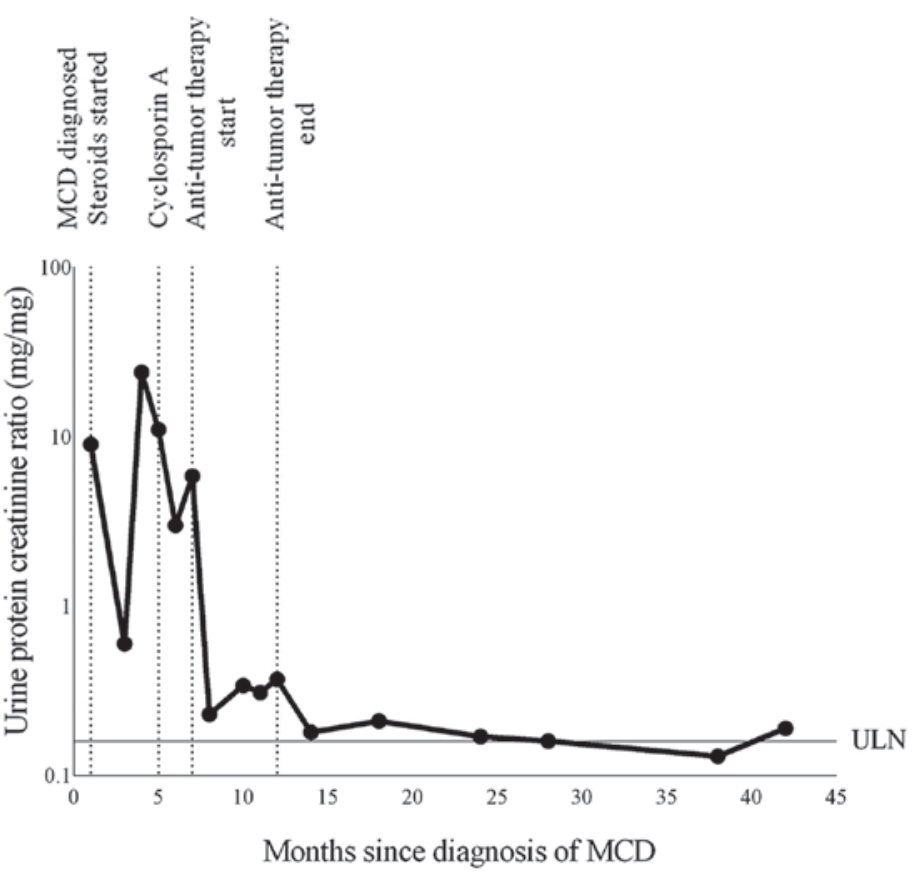

Figure 1. Trends in urine protein creatinine ratio (logarithmic 10 scale) following treatment with oral steroids, cyclosporine and tumor-directed therapy. MCD, minimal change disease; ULN, upper limit of normal.

Pre-treatment
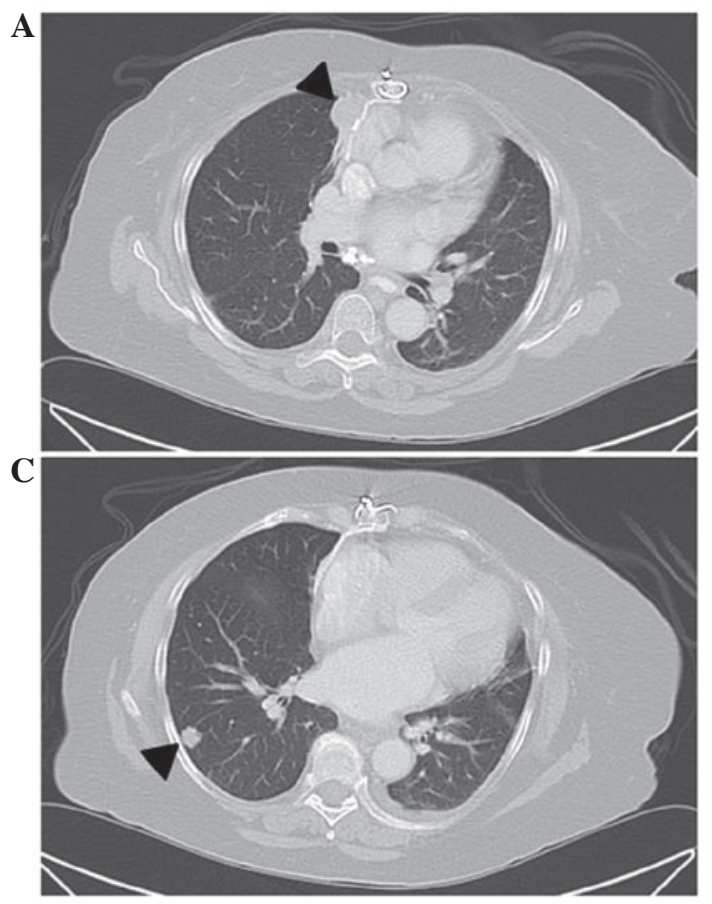

Post-treatment

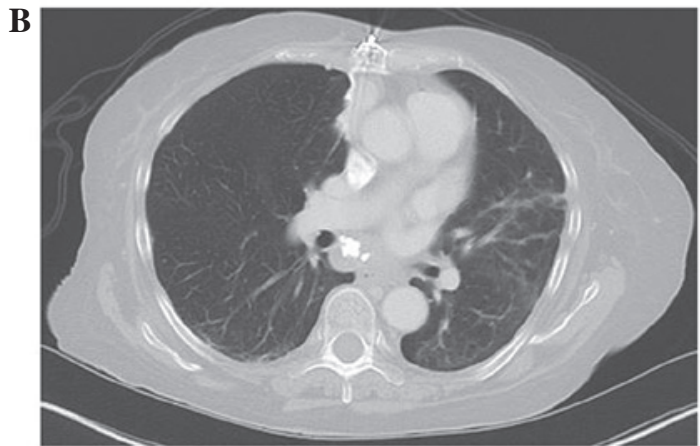

$\mathbf{D}$

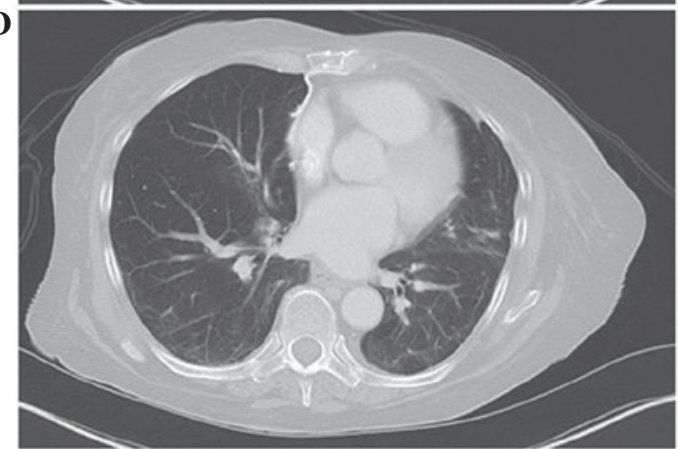

Figure 2. Representative axial computed tomography images of recurrent thymoma (A and C) prior to and (B and D) following treatment. Complete disappearance of (A) a right paracardiac mass and (C) a right lung nodule (indicated by arrowheads) was observed after six cycles of systemic antitumor therapy.

pulmonary nodules (Fig. 2). After obtaining written informed consent, the patient was enrolled in a phase I/II clinical trial (no. NCT01100944) that was approved by the Institutional Review Board of the National Cancer Institute (Bethesda, MD, USA). The patient was treated with 6 cycles of the histone deacetylase inhibitor, belinostat $\left[250 \mathrm{mg} / \mathrm{m}^{2}\right.$ administered as four consecutive 12-h continuous intravenous infusions (CIVI), starting on day 1 of a 21-day cycle], in combination with cisplatin [50 $\mathrm{mg} / \mathrm{m}^{2}$ intravenous (i.v.) on day 2], doxorubicin $\left(25 \mathrm{mg} / \mathrm{m}^{2}\right.$ i.v. once daily on days 2 and 3) and cyclophosphamide $\left(500 \mathrm{mg} / \mathrm{m}^{2}\right.$ i.v. on day 3) (7). Immune cell subsets, including regulatory $\mathrm{T}$ (Treg) and Thelper (Th) cells, were evaluated using multiparameter flow cytometry on whole blood samples collected prior to treatment (C1D1pre), on days 2 and 3 of cycle 1 (C1D2 and 

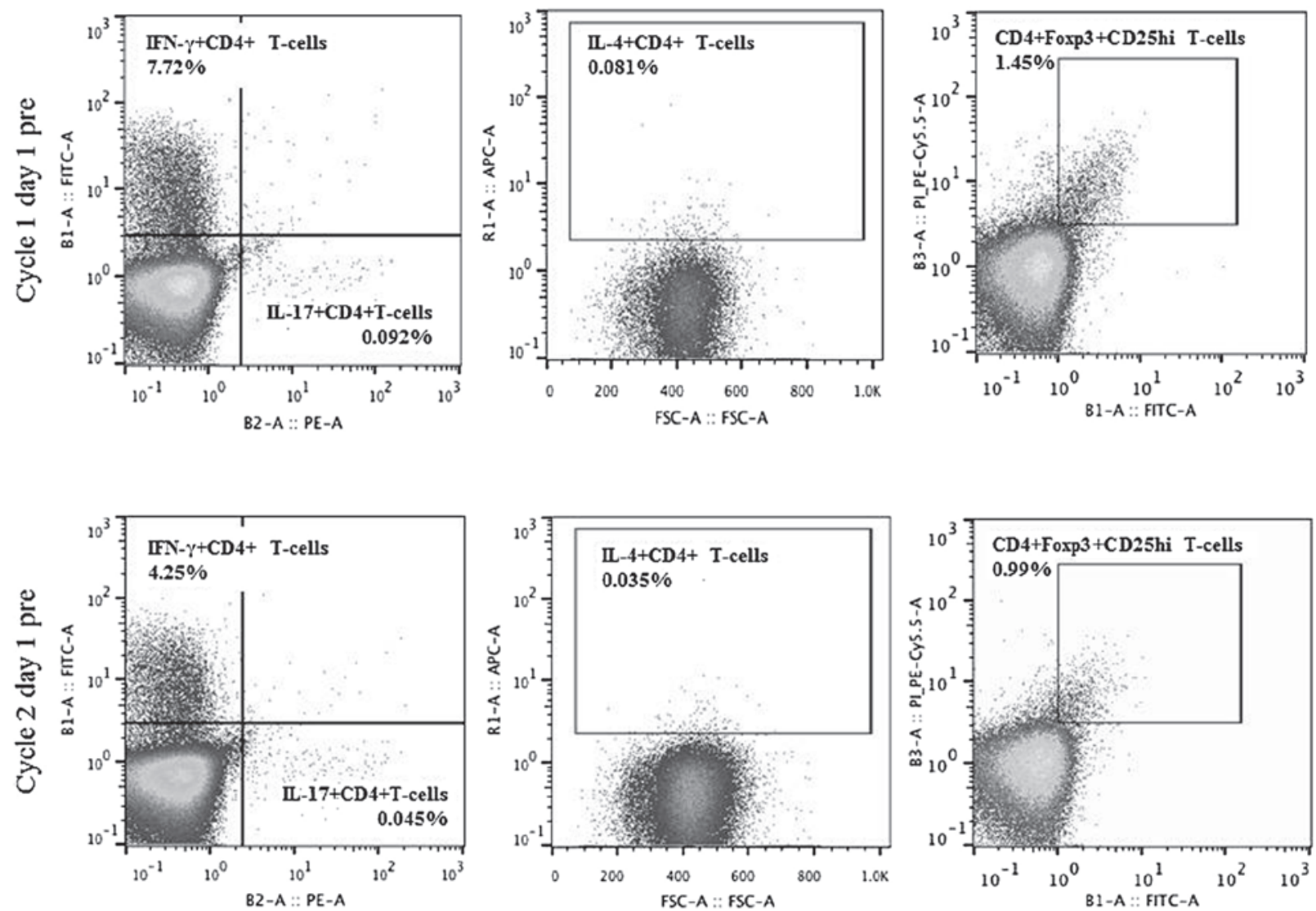

Figure 3. Changes in the population of Th1, Th2, Th17 and regulatory T cells in peripheral blood following systemic antitumor treatment. IFN- $\gamma$, IL-4 and IL-17 single-positive cells among $\mathrm{CD}^{+}{ }^{+} \mathrm{CD} 4^{+} \mathrm{T}$-cells were defined as Th1, Th2 and Th17, respectively. $\mathrm{CD} 4{ }^{+} \mathrm{CD} 25^{\text {high }}$ Foxp $3^{+}$cells were defined as regulatory $\mathrm{T}$ cells. Blood samples were collected at baseline (C1D1pre) and after 1 cycle of treatment (C2D1pre). Th, T helper; IFN, interferon; IL, interleukin.

C1D3, respectively) and prior to treatment on day 1 of cycle 2 (C2D1pre). The population of Th1, Th2, Th17 and Treg cells decreased on C2D1pre when compared with the population on C1D1pre, with fold-changes of $0.55,0.43,0.49$ and 0.69 , respectively (Fig. 3). A reduction in the Th17/Treg ratio was also observed, whereas the Th1/Th2 ratio increased (C2D1pre fold-change, 0.71 and 1.29, respectively). Proteinuria resolved following one cycle, and the urine protein-creatinine ratio was $0.15 \mathrm{mg} / \mathrm{mg}$. Cyclosporine and prednisone were discontinued within 4 months and a complete radiological response was observed within 6 months (Fig. 2). The reduction in proteinuria was durable as demonstrated by a urine protein-creatinine ratio of $0.19 \mathrm{mg} / \mathrm{mg} 30$ months after completion of the treatment (Fig. 1).

\section{Discussion}

To the best of our knowledge, the present study is the first comprehensive analysis of changes in T-cell subsets in response to tumor-directed treatment in thymoma-associated MCD. An improvement in proteinuria coincided with a reduction in the Th17/Treg ratio and an increase in the Th1/Th2 ratio.

A high Th17/Treg ratio has been previously observed in association with increased proteinuria and decreased serum albumin levels in primary MCD (8). Corticosteroid therapy has been demonstrated to result in a reduction in proteinuria and normalization of the Th17/Treg ratio due to a decrease in the Th17 cell population and an increase in the Treg cell population (8). In the current study, a reduction in the Th17/Treg ratio and a significant decline in proteinuria were observed following antitumor treatment, thus suggesting that a high Th17/Treg state may also play a role in the development of thymoma-associated MCD. However, in contrast to primary MCD, a decline in Treg cells accompanied by a sharper decline in Th17 cells, resulting in a reduction of the Th17/Treg ratio, was observed. These changes were also accompanied by a reduction in the tumor size. The population of Treg cells is frequently increased in the presence of a tumor and these cells play a critical role in the suppression of antitumor immune responses (9). In idiopathic nephrotic syndrome, induction of Treg cells is considered to represent a potential novel therapeutic strategy (10); however, in the present study, we hypothesize that a reduction in Treg cells by effective antitumor therapy plays an important role in the restoration of antitumor immune responses and results in an indirect improvement in thymoma-associated MCD.

Previous studies using the Buffalo/Mna rat model of spontaneous thymoma and nephrotic syndrome have demonstrated that polarization of the immune response toward a Th2 profile is associated with the development of glomerulonephritis $(11,12)$. To the best of our knowledge, the current study is the first to demonstrate a shift away from Th2 cells and an increase in the Th1/Th2 ratio with an associated reduction in proteinuria in a patient with thymoma-associated MCD. These observations are suggestive of an improvement in underlying T-cell dysfunction following administration of systemic antitumor therapy. Although these results require 
further validation, they may help in understanding the pathophysiologic mechanisms underlying thymoma-associated glomerulonephritis, and provide a rationale for rapid initiation of tumor-directed therapy. The benefits of this approach in controlling thymoma-associated paraneoplastic syndromes have been described previously (13).

In conclusion, the present study describes a case of relapsed, thymoma-associated MCD with a durable reduction in proteinuria following successful treatment of thymoma, accompanied by changes in immune cell subsets in peripheral blood. Early administration of antitumor therapy should be considered in such cases.

\section{Acknowledgements}

The authors would like to acknowledge the Intramural Research Program of the NIH, the National Cancer Institute and the Center for Cancer Research for their support of this study.

\section{References}

1. Nachman PH, Jennette JC and Falk R: Primary glomerular disease. In: The Kidney. Taal MW, Chertow GM, Marsden PA, Skorecki K, Yu ASL and Brenner BM (eds). Elsevier, Philadelphia, PA, pp1100-1191, 2012.

2. Hogan J and Radhakrishnan J: The treatment of minimal change disease in adults. J Am Soc Nephrol 24: 702-711, 2013.

3. Chugh SS, Clement LC and Macé C: New insights into human minimal change disease: Lessons from animal models. Am J Kidney Dis 59: 284-292, 2012.
4. Lien YH and Lai LW: Pathogenesis, diagnosis and management of paraneoplastic glomerulonephritis. Nat Rev Nephrol 7: 85-95, 2011.

5. Koga K, Matsuno Y, Noguchi M, Mukai K, Asamura H, Goya T, and Shimosato Y: A review of 79 thymomas: Modification of the staging system and reappraisal of conventional division into invasive and non-invasive thymoma. Pathol Int 44: 359-367, 1994.

6. Travis WD, Brambilla E, Müller-Hermelink HK and Harris CC (eds): Tumors of the thymus. In: World Health Organization Classification of Tumors: Pathology and Genetics of Tumors of the Lung, Pleura, Thymus and Heart. IARC Press, Lyon, France pp146-248, 2004.

7. Thomas A, Rajan A, Szabo E, Tomita Y, et al: A phase I/II trial of belinostat in combination with cisplatin, doxorubicin, and cyclophosphamide in thymic epithelial tumors: A clinical and translational study. Clin Cancer Res 20: 5392-5402, 2014.

8. Liu LL, Qin Y, Cai JF, Wang HY, Tao JL, Li H, Chen LM, Li MX, Li XM and Li XW: Th17/Treg imbalance in adult patients with minimal change nephrotic syndrome. Clin Immunol 139: 314-320, 2011.

9. Nishikawa $\mathrm{H}$ and Sakaguchi S: Regulatory $\mathrm{T}$ cells in cancer immunotherapy. Curr Opin Immunol 27: 1-7, 2014.

10. Le Berre L, Bruneau S, Naulet J, Renaudin K, Buzelin F, Usal C, Smit H, Condamine T, Soulillou JP and Dantal J: Induction of $\mathrm{T}$ regulatory cells attenuates idiopathic nephrotic syndrome. J Am Soc Nephrol 20: 57-67, 2009.

11. Lien YH and Lai LW: Pathogenesis, diagnosis and management of paraneoplastic glomerulonephritis. Nat Rev Nephrol 7: 85-95, 2011

12. Le Berre L, Hervé C, Buzelin F, Usal C, Soulillou JP and Dantal J: Renal macrophage activation and Th2 polarization precedes the development of nephrotic syndrome in Buffalo/Mna rats. Kidney Int 68: 2079-2090, 2005.

13. Rajan A, Kotlyar D and Giaccone G: Acute autoimmune hepatitis, myositis, and myasthenic crisis in a patient with thymoma. J Thorac Oncol 8: e87-e88, 2013. 